\title{
A Morphological Study of Placenta in Normal and Diabetic Pregnancies and Its Foetal Implications
}

\author{
Lisha S. Raj ${ }^{1 *}$, Sheela Vasudevan ${ }^{2}$ and Jayalekshmi ${ }^{1}$ \\ ${ }^{1}$ Department of Pathology, Dr. SMCSI Medical College, Trivandrum, India \\ ${ }^{2}$ Department of Pathology, Sree Gokulam Medical Collge, Trivandrum, India
}

\begin{abstract}
Background: The placenta is a foeto-maternal organ that separates the maternal and foetal circulations and plays a key metabolic role in pregnancy. Placental changes are directly proportional to the disease duration and its severity. In addition foetal outcome is also adversely influenced by pathological changes in placenta. . In the present study the focus is to bring out placental changes specifically associated with diabetes
\end{abstract}

Methods: The placentae for the study were obtained from the in- patients of department ofObstetrics and Gynaecology, SreeGokulam Medical College and Research Foundation, Venjaramoodu. 100 placentae were collected for the study. Among these 50 placentae were from mothers with uneventful antenatal period comprising the normal group and 50 placentae from mothers with GDM classified as GDM group.A descriptive study was done to assess the morphology of placentae in Gestational diabetes mellitus and to compare the same with that of normal pregnancies. The parameters included both gross and microscopy.

Result: The morphometric parameters and gross observations like hematoma and calcification were increased in GDM. Microscopic findings like Syncytial knots, fibrinoid necrosis, Cytotrophoblastic proliferation, basement membrane thickening, obliterative endarteritis, Villous vascularity and villous stromal fibrosis $(\mathrm{p}<0.001)$ were significantly increased in GDM group. Significant percentage of placentae with more than $5 \%$ of immature villi was seen in GDM group. The birth weight of babies was increased in GDM group mothers compared to normal group

Conclusion: Diabetes in pregnancy has a profound impact on both the placenta and the foetus. Both the villous and stromal parameters are altered by the high glycemic level.

Keywords: Placentae. Normal pregnancy.. Gestational diabetes mellitus. Morphology. Foetus.

\section{Introduction}

In recent years placenta has drawn significant attention as a valuable indicator for maternal and foetal diseases. Many of the disorders of pregnancy which are associated with high perinatal morbidity and mortality are accompanied by gross pathological changes in placenta. ${ }^{1}$ Most obstetricians and pediatricians would agree that examination of placenta often helps to explain an abnormal neonatal outcome. ${ }^{2}$ Despite the understanding, great resistance still exists in performing placental examination routinely. Thus placenta has remained as a mysterious organ for many pathologists.

Gestational diabetes also has an adverse effect on foetus and mother. The severity and extent of diabetes in pregnancy corresponds to placental changes at term and show a number of variations in comparison to placentae of nondiabetic mothers. ${ }^{1,2}$ The diabetic environment may have profound effects on placental development and functions. Placentae tend to be heavier and exhibit immaturity of villi. Recently it was proposed that these specific effects critically depend on the period of gestation. Some placental alterations continue to occur despite improvements in maternal glycemic control over the last decade, indicating that hyperglycemia is not the only causal factor.

Even though the placental changes are more common in the above conditions mentioned, many are not specific. Hence it is necessary to correlate placental changes in diabetic pregnancy with that of normal pregnancy. In order to generalize the specific changes a descriptive study is carried out on a total of 100 placentae which include 50 each from normal and diabetic pregnancies. The foetal implications of the placental changes associated with diabetes is also within the scope of study for which the results are also presented.

The objectives of the study are to describe placental morphology in normal \& diabetic pregnancies and to compare the changes and correlate above findings with foetal outcome.

\section{Materials and Methods}

This is a Descriptive Study conducted in Department of Pathology, Sree Gokulam Medical College \& Research 
Foundation. Placenta of all pregnant women admitting to the labour room with gestational age $>34$ weeks $\&<42$ weeks is determined as study population. Minimum of 50 placenta of GDM cases admitted in the labour room and Equal number of placenta of normal delivery will be taken for Comparison. The sample size has been calculated as per the formula:

$N=\frac{Z_{\alpha}^{2} \times P \times(1-P)}{d^{2}}$

$\mathrm{N}$ is the sample size

$\mathrm{P}$ is the prevalence

$\mathrm{D}$ is $20 \%$ of $\mathrm{P}$, weighing factor for the probability

$\mathrm{Z} \alpha$ is the proportionality constant which in this case is 1.96 .

Placenta of Pregnant Women with Gestational Age of 32 42 Weeks of gestation with Gestational Diabetes Mellitus and uncomplicated antenatal period were taken for the study. The details of the study protocol were explained to the subjects and informed consent obtained. Relevant clinical history, examination findings \& investigation records obtained from the Obstetrics \& Gynaecology department. The placentae were collected immediately following delivery and washed in tap water and blotted to remove the blood clots. The shape of the placenta was assessed. The foetal surface was examined for color. The extra placental membranes were inspected for completeness, adherent blood clot, color transparency and insertion. The umbilical cord was measured and was examined for knots (true/ false) and color (normal/ green/ brown/ yellow or gray). Umbilical cord insertion was also determined. The membranes were trimmed and a strip was taken as "swiss roll" for histopathological examination. The cord was cut at about $4 \mathrm{cms}$ from its insertion. The number of blood vessels in the cut end was determined. The maternal surface of the placenta was inspected for completeness, adherent blood clots, appearance (pale/ congested or unremarkable), fibrin, calcification and infarction and percentage of parenchymal involvement. The whole placenta was left for fixation in $10 \%$ formalin for $24-48$ hours. After fixation the following variables were studied :
a. Weight of the placenta
b. Diameter of the placenta
c. Thickness of the placenta
d. Number \& Intactness of cotyledons
e. Diameter of cord

Later placenta was cut into vertical strips (bread loaf manner) of $0.5 \mathrm{cms}$ thickness and gross lesions were re- examined. Tissues for microscopic examination were taken from:-

i. Umbilical cord at placental junction and cut end.(2 bits)

ii. Strip of membrane roll.(1 bit)

iii. Both sides of umbilical cord insertion on placenta.(2 bits)

iv. Fibrotic area if present.

v. Infarcted area, if present.

vi. Any other gross lesions.

Tissue sections of $5 \mu \mathrm{m}$ thickness were cut from paraffin embedded blocks and stained by conventional haematoxylin and eosin stain. According to the findings special staining techniques like PAS for basement membrane thickening and Van Gieson for obliterative endarteritis \& stromal fibrosis were employed. Various histological features were quantified. For this one hundred terminal villi were counted in each sections Thus, in each placenta four hundred villi were counted and mean calculated. The mean number of villi showing syncytial knots, fibrinoid necrosis, basement membrane thickening and villous stromal fibrosis were determined. The gross and microscopic parameters studied are tabulated in Table 1.

Exclusion criteria: Placentae of women with Multiple pregnancy, Pregnancy Induced Hypertension, Rh negative pregnancy, pregnant women with heart disease, Placenta Previa, Pregnancy with torch and other infections and Pregnant women with autoimmune disorders were excluded from the study

In addition, birth weight, one minute and five minute APGAR score, term/preterm, still birth, meconium staining, NICU admission, congenital anomaly etc was recorded from baby notes.

Data Analysis: The data was arranged in Excel Sheet under suitable headings. The mean and standard deviation or incidence was calculated for different parameters. They were statistically analyzed using Chi square test for qualitative variables and student $t$ test for quantitative variables. This was done using Microsoft Excel and SPSS. In all the tests ' $p$ ' value less than 0.05 was taken to be statistically significant and value less than 0.01 was taken as highly significant.

\section{Result}

The section presents the results and interpretation of the 100 placentae which includes 50 each from mothers with uncomplicated pregnancies - considered as control group and from mothers with gestational diabetes mellitus - 


\begin{tabular}{|c|c|c|}
\hline \multicolumn{3}{|c|}{ Gross parameters } \\
\hline Parameter & Classification & Remarks \\
\hline Calcification & Grading & $1+, 2+, 3+$ visual assessment \\
\hline Infarction & Present/absent & $>5 \%$ of parenchyma - present \\
\hline Hematoma & Grading & $\begin{array}{l}\text { Marginal, } 2 \text { - Retroplacental } \\
\text { - subchorinic }\end{array}$ \\
\hline Sub chorionic fibrin deposition & Grading & $1+, 2+, 3+$ based on visual assessment \\
\hline \multicolumn{3}{|c|}{ Microscopic parameters } \\
\hline Syncytial knots & Grading & $<30 \%, 30 \%-50 \%,>50 \%$ \\
\hline Fibrinoid necrosis & Grading & $\begin{array}{l}<3 \%, 3 \%-10 \% \&>10 \% \text { based on villi showing fibroid } \\
\text { necrosis }\end{array}$ \\
\hline Fibrotic villi & Present/absent & $<6 \%$ - absent, $>6 \%$ - present \\
\hline Cytotrophoblastic proliferation & Grading & $\begin{array}{l}<20 \%, 20 \%-40 \%,>40 \% \text { based on area of trophoblastic } \\
\text { hyperplasia }\end{array}$ \\
\hline Basement membrane thickening & Present/absent & $\begin{array}{l}<3 \%-\text { absent } \\
>3 \% \text { - present }\end{array}$ \\
\hline Obliterative endarteritis & Grading & $\begin{array}{l}1 \text { - mild, } 2 \text { - moderate, } \\
3 \text { - severe }\end{array}$ \\
\hline Villous vascularity & Decreased/Increased & $\begin{array}{l}<2 \text { vessels / villi - Decreased } \\
>6 \text { vessels / villi - increased }\end{array}$ \\
\hline Immature villi & Present/absent & $\begin{array}{l}<5 \%-\text { absent } \\
>5 \% \text { - present }\end{array}$ \\
\hline
\end{tabular}

considered GDM group. Majority of patients were in the age group of $20-25$ years, but it ranges from $18-37$ years. There is no statistically significant difference between the age in the two groups. Among the 100 pregnant women 59 were multiparous and 41 were primigravida. There is no statistically significant difference of parity between the two groups. Majority of patients delivered at $37-39$ weeks, with $54 \%$ of women with GDM delivering before 38 weeks and $46 \%$ of normal pregnancies terminating at 38 -39 weeks. The comparison of placental weight, thickness and diameter is given in Table 2. The gross and microscopic parameters analyzed are given in Table 3 .

The placental weight significantly increased in GDM when compared with that of normal pregnancies. It ranges from 400gms - 1100gms (mean 594gms) in GDM pregnancies and $300-800$ (mean 524gms) in normal pregnancies. $p$ value -0.000

Gross picture of GDM placenta with hematoma is given in Fig 1a. The photo micrographs of few microscopic parameters are also given :- Hematoma with Infarction (Fig 1b), Obliterative endarteritis (Fig 1c, 1d), villous vascularity (Fig 2a), Immature Villi (Fig 2b), Basement Membrane thickness (Fig 2c, 2d).

FOETAL PARAMETERS: GDM pregnancy showed statistically significant increase in birth weight of baby compared to normal group. Birth weight of more than $3.5 \mathrm{Kg}$ was seen in $28 \%$ of the GDM group compared to $6 \%$ in normal group.( $\mathrm{p}$ value -0.025$)$. No significant difference in APGAR Score noted in the two groups. APGAR of 9 at 1 min was noted in $90 \%$ of babies in GDM group and $92 \%$ of babies in Normal group. Rest $10 \%$ of babies in GDM group exhibit an APGAR of 9 at $5 \mathrm{~min}$. Among the normal group 3 babies have an APGAR score of 9 at 5 min and for one baby the score was $5-8$ at $1 \mathrm{~min}$. The comparison of baby weight is given in figure 3

NICU admission of babies is significantly high in GDM $(66 \%)$ when compared with Normal group (10\%). P value -0.000 . The mean of fetoplacental ratio observed were 5.5 in GDM group and 5.7 in normal group. But the marginal variation of fetoplacental ratio in normal and GDM group is not statistically significant in the present study.

\section{Discussion}

In the study on placenta Fox in 1997, the importance of analyzing the placental pathology quantitatively is stressed upon. ${ }^{1}$ The changes are considered pathological when the extent of involvement is greater than normal. In the present study a total of 100 placentae were included; 50 each from mothers with uncomplicated pregnancies considered normal group, and gestational diabetes mellitus -considered GDM group. The gross and microscopic 
Table 2: Placental Morphometry.

\begin{tabular}{|c|c|c|c|c|}
\hline \multicolumn{5}{|c|}{ Weight of placenta } \\
\hline \multirow[t]{2}{*}{ Weight (gms) } & \multicolumn{2}{|l|}{ GDM } & \multicolumn{2}{|l|}{ Normal } \\
\hline & Count & Percent & Count & Percent \\
\hline $200-400$ & 0 & 0.0 & 7 & 14.0 \\
\hline $400-600$ & 21 & 42 & 35 & 70 \\
\hline $600-800$ & 26 & 52 & 8 & 16 \\
\hline$>800$ & 3 & 6 & 0 & 0.0 \\
\hline Mean weight & \multicolumn{2}{|l|}{$594 \mathrm{gms}$} & \multicolumn{2}{|c|}{$524 \mathrm{gms}$} \\
\hline \multicolumn{5}{|c|}{ Diameter of placenta } \\
\hline Mean diameter & \multicolumn{2}{|l|}{$15.3 \mathrm{~cm}$} & \multicolumn{2}{|l|}{$14 \mathrm{~cm}$} \\
\hline \multicolumn{5}{|c|}{ Thickness of placenta } \\
\hline Mean thickness & \multicolumn{2}{|l|}{$5.2 \mathrm{~cm}$} & \multicolumn{2}{|l|}{$4.2 \mathrm{~cm}$} \\
\hline
\end{tabular}

Table 3

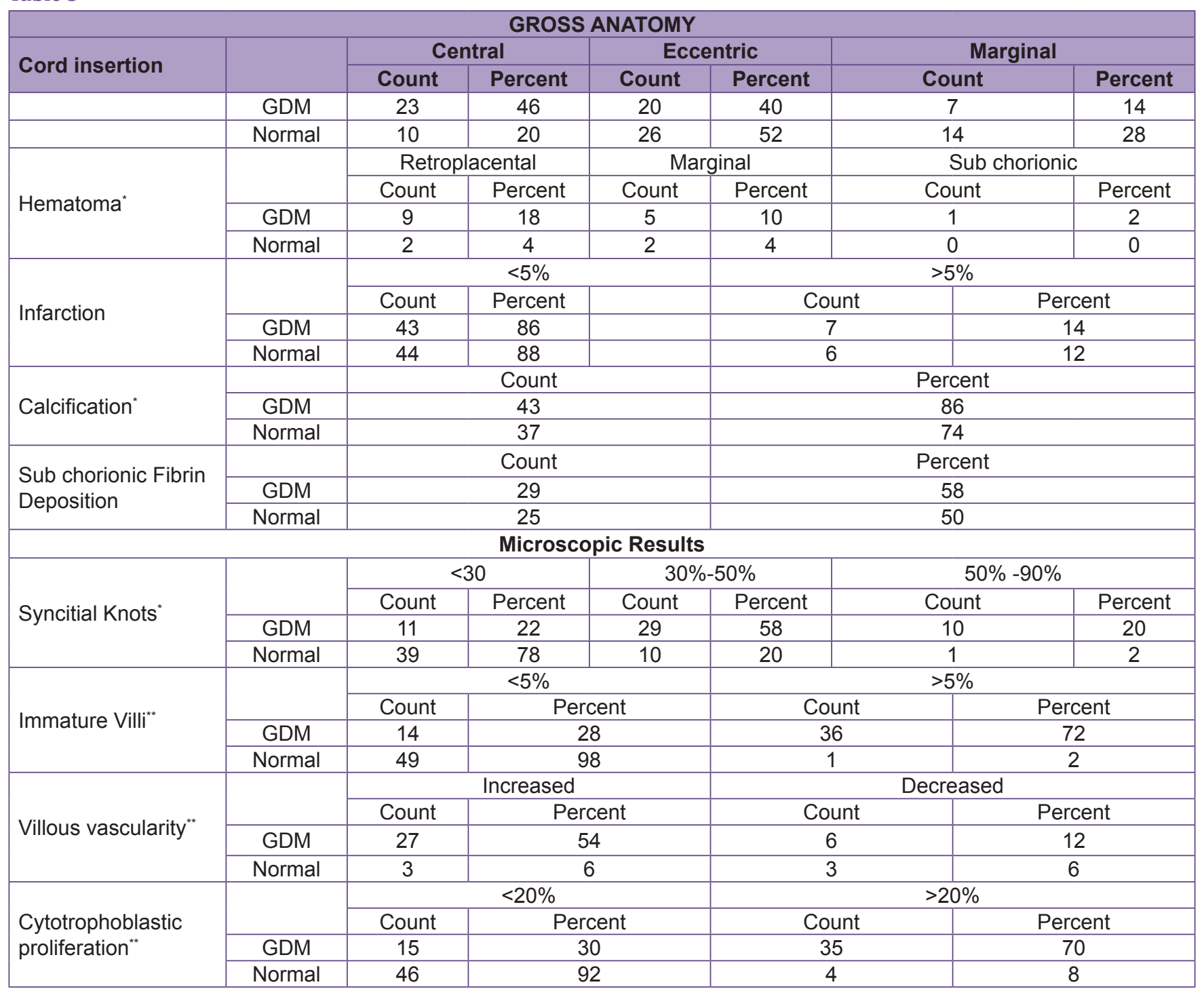




\begin{tabular}{|c|c|c|c|c|c|c|c|}
\hline \multicolumn{7}{|c|}{ GROSS ANATOMY } & \\
\hline \multirow{2}{*}{ Cord insertion } & & \multicolumn{2}{|c|}{ Central } & \multicolumn{2}{|c|}{ Eccentric } & \multicolumn{2}{|c|}{ Marginal } \\
\hline & & Count & Percent & Count & Percent & & Percent \\
\hline \multirow{4}{*}{$\begin{array}{l}\text { Basement } \\
\text { membrane } \\
\text { thickening }\end{array}$} & & \multicolumn{3}{|c|}{$<3 \%$} & \multicolumn{3}{|c|}{$>3 \%$} \\
\hline & & Count & \multicolumn{2}{|c|}{ Percent } & \multicolumn{2}{|c|}{ Count } & Percent \\
\hline & GDM & 21 & \multicolumn{2}{|c|}{42} & \multicolumn{2}{|c|}{29} & 58 \\
\hline & Normal & 46 & \multicolumn{2}{|c|}{92} & \multicolumn{2}{|c|}{4} & 8 \\
\hline \multirow{4}{*}{$\begin{array}{l}\text { Obliterative } \\
\text { endarteritis }\end{array}$} & & \multicolumn{3}{|c|}{ Mild } & \multicolumn{3}{|c|}{ Moderate + Extreme } \\
\hline & & Count & \multicolumn{2}{|c|}{ Percent } & \multicolumn{2}{|c|}{ Count } & Percent \\
\hline & GDM & 23 & \multicolumn{2}{|c|}{46} & \multirow{2}{*}{\multicolumn{2}{|c|}{$\frac{15}{1}$}} & 30 \\
\hline & Normal & 10 & & & & & 2 \\
\hline \multirow{4}{*}{ Fibrinoid necrosis" } & & \multicolumn{3}{|c|}{$3 \%-10 \%$} & \multicolumn{3}{|c|}{$>10 \%$} \\
\hline & & Count & & & \multicolumn{2}{|c|}{ Count } & Percent \\
\hline & GDM & 24 & \multicolumn{2}{|c|}{48} & \multicolumn{2}{|c|}{17} & 34 \\
\hline & Normal & 20 & \multicolumn{2}{|c|}{40} & & 2 & 4 \\
\hline & & & $<6 \%$ & & & & \\
\hline Villous stromal & & Count & & & & & Percent \\
\hline fibrosis $^{* *}$ & GDM & 24 & & & & & 52 \\
\hline & Normal & 45 & & & & 5 & 10 \\
\hline
\end{tabular}
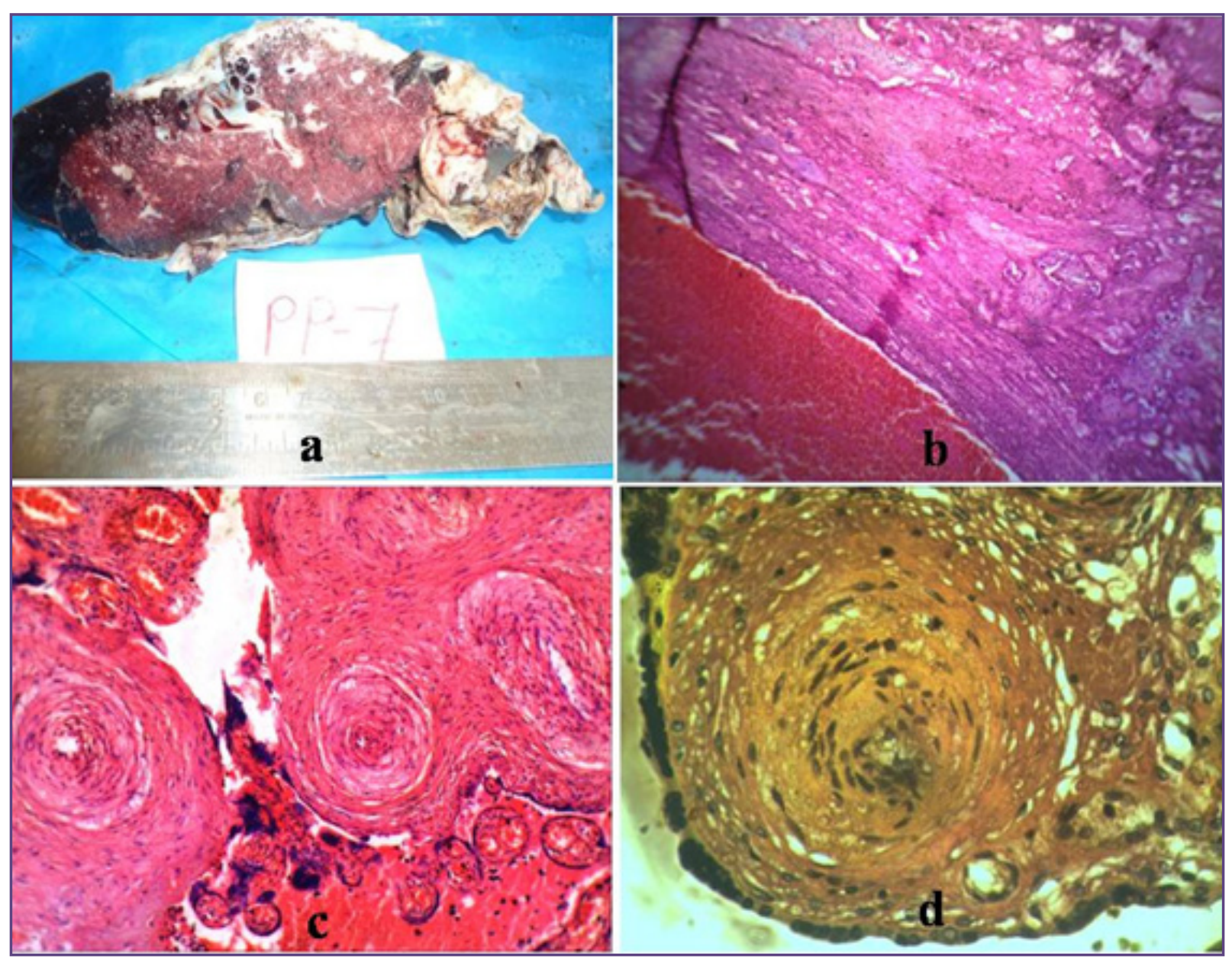

Fig. 1 a) Gross picture of Placenta with Hematoma in GDM. b) Hematoma with infarction in GDM Placenta (H\&E 10x) c) Villi of GDM placenta showing obliterative endarteritis (H\&E 10X) d) Villi of GDM placenta showing obliterative endarteritis (Van Gieson 40X). 


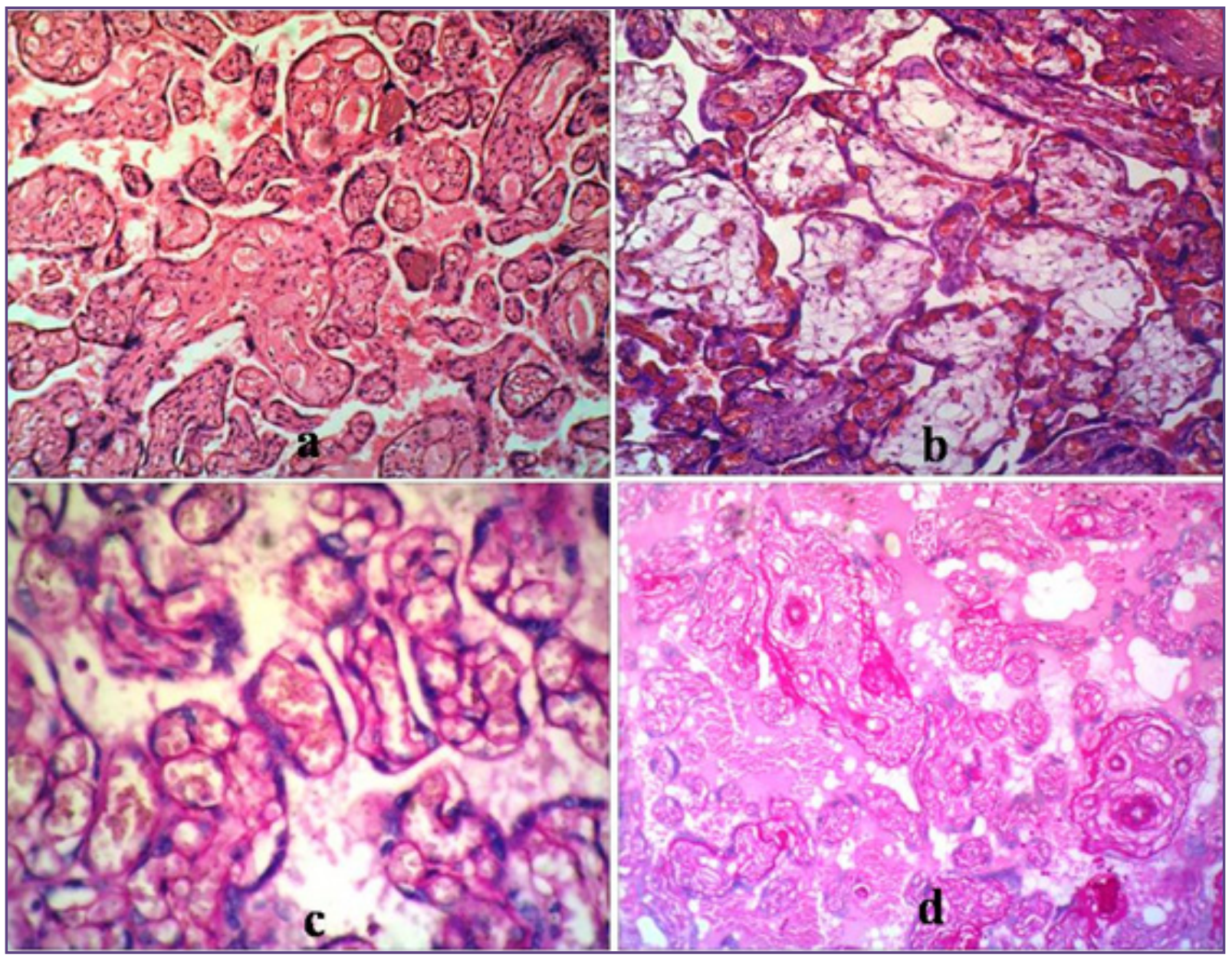

Fig. 2: a) Increased vascularity in GDM placenta (H\&E 10X). b) Immature Villi in GDM pregnancy (H\&E 40X) c) Villi showing normal basement membrane thickness (PAS 40X) in normal Placenta d) Villi showing increased basement membrane thickness in placenta of GDM group (PAS 10X).

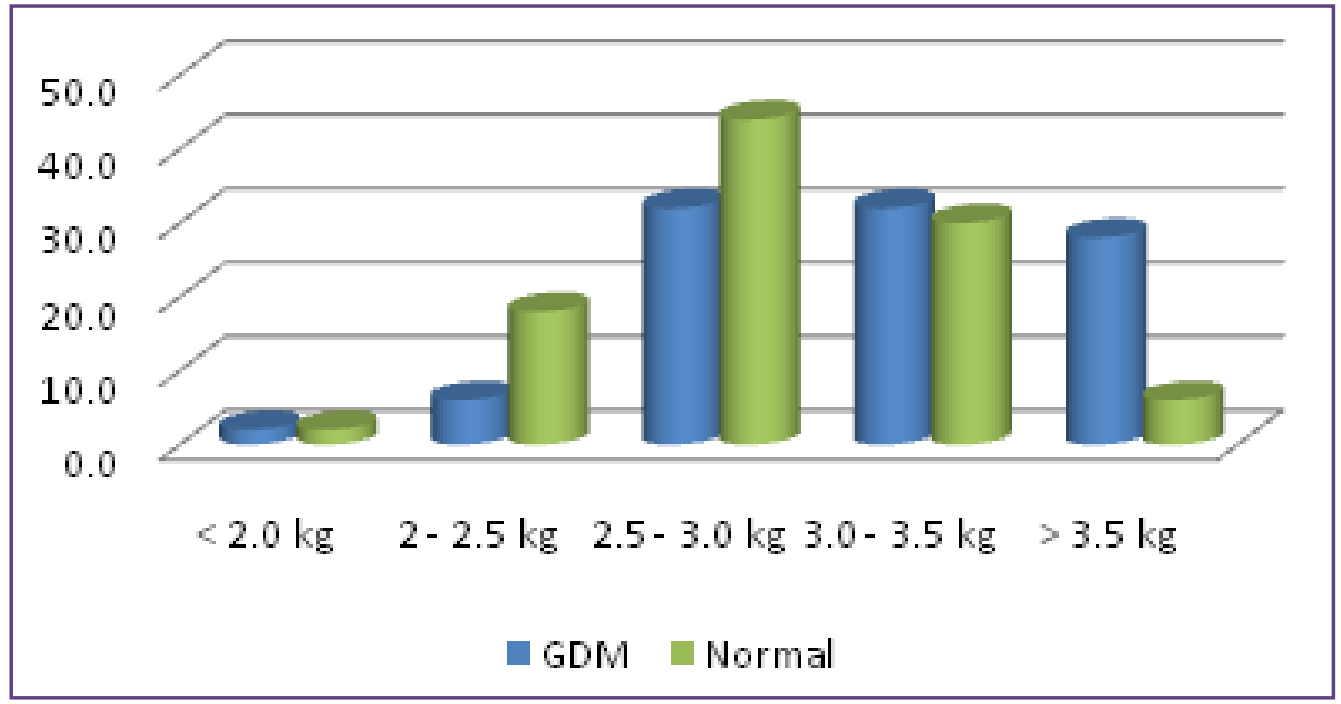

Fig. 3: Comparison of weight of baby based on group. 
lesions were quantified and compared between the two groups and results correlated were with the foetal outcome. There is no statistically significant difference between the age \& parity in the two groups as in the study done by Goerge Daskalakis et. al. ${ }^{6}$

Among morphometric parameters, weight of placenta is of considerable importance as it is related to villous area and foetal metabolism. In the present study placental weight was significantly increased in GDM when compared with normal pregnancies. Placenta of pregnancies complicated by GDM showed increased mean diameter and thickness when compared to placenta of normal pregnancies which correlates with studies of. Soma Saha. ${ }^{7}$

In the gross anatomy, three types of attachment were noted; central, marginal and eccentric based on the distance from center and periphery to the cord attachment site. Majority of the placenta showed eccentric insertion of cord as with Ranjana Verma et. al. ${ }^{8}$ Among the placenta of GDM complicating pregnancies central cord insertion is found to be statistically more prevalent p value of 0.016 . The presence of hematoma is significantly increased in pregnancies complicated by GDM and PIH ( $p$ value 0.005 ). Among the 19 placenta showing hematoma, 11 were retroplacental of which 9 (18\%) from GDM group, and 2 (4\%) from normal group. According to Bandana Das et. al in $1996^{1}$, the presence of retroplacental hematoma was associated with low APGAR score babies and larger hematoma was also associated with intrauterine foetal death. This is because a considerable portion of villi are acutely separated from the maternal utero placental circulation. No such occurrences were noted in the present study. Calcification is common in human placentae and is recognized as a normal part of maturation and ageing of this organ. The pathological maturation can lead to foetal growth restriction which is the second most common cause of perinatal death after prematurity. Pregnancies complicated by GDM showed significant increase in calcification $\mathrm{p}$ value 0.015 . $^{7}$

Microscopic study revealed increased syncytial knots in placentae from GDM group and is highly significant with a $p$ value of 0.000 , correlated with study by Vineeta Tewari et. al. ${ }^{10,8,7}$ GDM group showed increase in fibrinoid necrosis when compared to normal group ( $p$ value o.ooo). This increased fibrinoid necrosis in GDM is in accordance with ${ }^{8}$ and George Daskalakis et. al. ${ }^{6}$ According to Shams et. al. (2012) there was significantly greater fibrinoid necrosis in placenta from mothers having diabetes and hypertension. ${ }^{11}$ Cytotrophoblastic proliferation was significantly increased in GDM group when compared with normal with a $\mathrm{p}$ value of 0.000 . This is in accordance with studies conducted by Majumdar et. al. ${ }^{12,7}$ The comparison of villi showing
Basement membrane thickening $>3 \%$ showed significant increase of the finding in GDM group(p value 0.000$)$, in accordance with $^{10,14,7}$. Increases in basement membrane thickening were demonstrated by PAS staining. Hypoxic conditions in pregnancy leads to loss of large number of parenchymal cells and deposition of fibrous tissue. The fibrous tissue synthesized by fibroblasts of stroma is responsible for the basement membrane thickening. Studies revealed that diabetic pregnancies show an increased incidence of vascular pathological changes. Such changes can affect the arteries and veins in stem villi of different sizes, corresponding to peripheral vessels. The lesions affect the fetoplacental circulation. ${ }^{1}$ In the present study the increased vascularity is found to be significant when compared to normal group ( $p$ value 0.000 ) and is in accordance with Lal Baksh Khaskhelli et. $\mathrm{Al}^{14}$, Ranjana Verma et. $\mathrm{Al}^{8}$ and George Daskalakis et. al. ${ }^{6}$ More than 5\% of immature villi was seen in large number (72\%) in GDM group when compared to $(2 \%)$ of Normal group. This finding is found to be statistically significant. ( $\mathrm{p}$ value 0.000 ), in accordance with findings of Evers, Riza and Lal Baksh Khaskhelli ${ }^{14}$

Stromal pathology noted include fibrosis, calcification and endothelial proliferation. Increased number of villi showing stromal fibrosis in GDM when compared to normal group is found to be statistically significant. (0.000). In the study conducted by Vineeta Tewari etal. ${ }^{10}$ Increased villous stromal fibrosis in 18 out of $30(60 \%)$ of GDM placentae is noted. Presence of obliterative endarteritis was significantly increased in GDM group compared with normal. p value 0.000 Extensive obliterative endarteritis were seen none in normal and GDM group. ${ }^{7}$

Foetal Parameters: GDM pregnancy showed statistically significant( $\mathrm{p}$ value -0.025 increase in birth weight (mean $3.17 \mathrm{~kg}$ ) of baby compared to normal group (mean $2.9 \mathrm{~kg}$ ). Birth weight of more than $3.5 \mathrm{Kg}$ was seen in $28 \%$ of the GDM group compared to $6 \%$ in normal group. In ${ }^{7}$ mean weight of babies of GDM pregnancies was $3.9 \mathrm{~kg}$ which was greater than mean weight of $2.53 \mathrm{~kg}$ in normal pregnancies. ${ }^{7}$ The pathophysiology behind weight gain include compensatory hyperplasia due to foetal macrosomia, which in turn result from reactionary hyperglycemia in foetuses of diabetic mothers. No significant difference in APGAR Score noted in the two groups. NICU admission of babies are significantly high in GDM(66\%) when compared with Normal group (10\%) P value - 0.000. Mean foeto-placental weight ratio observed in the present study were 5.5 in GDM group and 5.7 in normal group. The marginal variation of foeto-placental weight ratio in GDM is not statistically significant in the present study. Significant results were 
obtained in several other studies. In the study Soma Saha et. $\mathrm{Al}^{7}$ claims increased foeto-placental weight ratio. The increased placental weight was proportionally greater than the increased foetal weight in the diabetic group. This leads to a decreased foeto-placental weight ratio because much placental growth occurs in the first half of gestation, well before GDM diagnosis and the start of treatment.

\section{Conclusion}

The present study was undertaken to evaluate quantitatively the histopathologic changes in placentae obtained from Gestational diabetes (GDM) mothers and compare them with placentae of mothers with uneventful antenatal period. The morphometric parameters viz. placental weight, placental diameter and placental thickness were increased in GDM group in comparison to normal group. Gross observations like hematoma and calcification were increased in both GDM group. Significant microscopic changes were observed in placentae of GDM group as compared to controls viz. increased syncytial knots, fibrinoid necrosis, cytotrophoblastic proliferation, basement membrane thickening, obliterative endarteritis ,increased villous vascularity and villous stromal fibrosis $(p<0.001)$. Significant percentage of placentae with more than $5 \%$ of immature villi were seen in GDM group when compared to normal group. The birth weight of babies were increased in GDM group mothers compared to normal group. The incidence of NICU admission was significantly increased in GDM.

\section{Reference}

1. Damjanov I, Linder J. Anderson's Pathology, 10th Edition, Elsevier.

2. Robboy S J et.al. Robboy's Pathology of the Female Reproductive Tract. 2nd Edition, Elsevier.

3. Marilza V C, Rudge, Cesar P, Debora C, Yuri K, Gustavo $\mathrm{N}$, CibeleVC. Histopathological placental lesions in mild gestational hyperglycemic and diabetic women. Diabetology \& Metabolic Syndrome. 2011.
4. Tewari V, Tewari A, Bhardwaj N. Histological and histochemical changes in placenta of diabetic pregnant females and its comparision with normal placenta. Asian Pacific Journal of Tropical Disease. 2011.

5. Fox H. Ageing of placenta. Arch Dis Child Foetal Neonatal. 1997; 77: 171-175.

6. Daskalakis G, Marinopoulos S, Krielesi V, Papapanagiotou A, Papantoniou N, Esogitis S. Placental pathology in women with gestational diabetes. Acta Obstetricia et Gynecologica. 2008; 87: 403-407.

7. Saha S, Biswas S, Mitra D, Adhikari A, Saha C. Histologic and morphometric study of human placenta in gestational diabetes mellitus. IJAE.2014; 1(119): $1-9$.

8. Verma R, Mishra S, Kaul J. Cellular Changes in the Placenta in Pregnancies Complicated with Diabetes. Int. J. Morphol.2010; 28(1):259-264.

9. Bandana D, Dutta D, Chakraborthy D S, Nath P. Placental morphology in hypertensive disorders of pregnancy and its correlation with foetal outcome. Journal of Obstet and Gynecol India. 1996; 46(1):40-46 .

10. Tewari V, Tewari A, Bhardwaj N. Histological and histochemical changes in placenta of diabetic pregnant females and its comparision with normal placenta. Asian Pacific Journal of Tropical Disease. 2011.

11. Shams F, Rafique M, Samoo NA, Irfan R. Fibrinoid Necrosis and Hyalinization Observed in Normal, Diabetic and Hypertensive Placentae. Journal of the College of Physicians and Surgeons. 2012; Vol. 22 (12): 769-772.

12. Majumdar S, Dasgupta $\mathrm{H}$, Bhattacharya $\mathrm{K}$, Bhattacharya A. A Study of Placenta In Normal and Hypertensive Pregnancies. J Anat Soc India.2005;54(2):1-9.

13. Maternal diseases complicating pregnancy: diabetes, tumors, preeclampsia, lupus anticoagulant. In: Benirschke K, Kaufmann P (eds). Pathology of the Human Placenta, 3rd edn. New York: Springer; 1995: 476-536.

14. Khaskhelli LB, Memon S, Goswami P, Bano S. Change in Normal Morphology of Placenta and Its Possible Effects on Foetal Outcome in Diabetic Mothers as Compared to NonDiabetic Mothers. JLUMHS. January-April 2013; 1(12).

*Corresponding author:

Dr. Lisha S. Raj, Postal Address: Dreams, TC 4/1542 (3), Pandit Colony, Kowdiar, Trivandrum-695003, INDIA

Phone: +919496465607

Email: lishasraj@gmail.com

Financial or other Competing Interests: None. 